\title{
A randomized double-blind placebo-controlled trial to investigate the effects of nasal calcitonin on bone microarchitecture measured by high-resolution peripheral quantitative computerized tomography in postmenopausal women - Study protocol

\author{
Laura Richert ${ }^{1}$, Brigitte Uebelhart ${ }^{1}$, Marc Engelhardt ${ }^{2}$, Moise Azria ${ }^{2}$ and \\ René Rizzoli*1
}

Address: ${ }^{1}$ Service of Bone Diseases, Department of Rehabilitation and Geriatrics, University Hospitals of Geneva, 24, Rue Micheli-du-Crest, 1211 Geneva 14, Switzerland and ${ }^{2}$ Novartis Pharma AG, 4002 Basel, Switzerland

Email: Laura Richert - laura.richert@isped.u-bordeaux2.fr; Brigitte Uebelhart - brigitte.uebelhart@hcuge.ch;

Marc Engelhardt - marc.engelhardt@ novartis.com; Moise Azria - moise.azria@novartis.com; René Rizzoli* - rene.rizzoli@hcuge.com

* Corresponding author

Published: 13 April 2008

Trials 2008, 9:19 doi:10.1186/1745-6215-9-19

This article is available from: http://www.trialsjournal.com/content/9/1/19

(c) 2008 Richert et al; licensee BioMed Central Ltd.

This is an Open Access article distributed under the terms of the Creative Commons Attribution License (http://creativecommons.org/licenses/by/2.0), which permits unrestricted use, distribution, and reproduction in any medium, provided the original work is properly cited.
Received: 13 December 2007

Accepted: 13 April 2008

\begin{abstract}
Background: Bone microarchitecture is a significant determinant of bone strength. So far, the assessment of bone microarchitecture has required bone biopsies, limiting its utilization in clinical practice to one single skeletal site. With the advance of high-resolution imaging techniques, noninvasive in vivo measurement of bone microarchitecture has recently become possible. This provides an opportunity to efficiently assess the effects of anti-osteoporotic therapies on bone microarchitecture. We therefore designed a protocol to investigate the effects of nasal salmon calcitonin, an inhibitor of osteoclast activity, on bone microarchitecture in postmenopausal women, comparing weight bearing and non-weight bearing skeletal sites.
\end{abstract}

Methods: One hundred postmenopausal women will be included in a randomized, placebocontrolled, double-blind trial comparing the effect of nasal salmon calcitonin (200 Ul/day) to placebo over two years. Bone microarchitecture at the distal radius and distal tibia will be determined yearly by high-resolution peripheral quantitative computerized tomography ( $\mathrm{P}-\mathrm{QCT}$ ) with a voxel size of $82 \mu \mathrm{m}$ and an irradiation of less than $5 \mu$ Sv. Serum markers of bone resorption and bone formation will be measured every 6 months. Safety and compliance will be assessed. Primary endpoint is the change in bone microarchitecture; secondary endpoint is the change in markers of bone turnover.

Hypothesis: The present study should provide new information on the mode of action of nasal calcitonin. We hypothezise that - compared to placebo - calcitonin impacts on microstructural parameters, with a possible difference between weight bearing and non-weight bearing bones.

Trial Registration: ClinicalTrials.gov NCT00372099 


\section{Background}

Osteoporosis is characterized by compromised bone strength and higher fragility, resulting in an increased fracture risk [1]. The measurement of areal bone mineral density (BMD) by dual-energy X-ray absorptiometry (DXA) is the most common way to measure bone mass and to assess fracture risk. Yet, bone strength is also determined by bone geometry, bone microarchitecture, bone remodelling and intrinsic tissue quality [2]. Thus, it is not surprising that BMD only partially explains the variance of strength and fracture risk [3]. It has been postulated that markers of bone remodelling determined in blood or urine could be independent predictors of fracture risk [4]. Assessment of changes in bone microarchitecture has been limited, since bone biopsies were necessary to investigate these parameters in the past. This technique is limited to a non-weight bearing bone such as the iliac crest. Today, high-resolution peripheral quantitative computerized tomography (p-QCT) allows an efficient and quantitative evaluation of bone microarchitecture $[5,6]$. It determines in three dimensions true volumetric density $\left(\mathrm{g} / \mathrm{cm}^{3}\right)$, and can directly assess trabecular thicknesses, trabecular separations, and trabecular number, and also provides additional geometric variables such as cross-sectional area (CSA) and information on structural pattern (rod vs. plate structure) with a relatively low irradiation, high accuracy and precision [7]. P-QCT thus provides a unique non-invasive opportunity to explore the effects of diseases on bone microarchitecture and of therapies intended to halt or reverse these changes [8].

Postmenopausal bone loss and fracture risk are associated with an imbalance in bone turnover. As a therapeutic agent, calcitonin binds to specific receptors on the osteoclasts and reduces their relative hyperactivity.

The formula of nasal calcitonin is well tolerated. The most common side effects consist of mild or moderate rhinitis symptoms, such as nasal congestion and sneezing, or nasal dryness $[9,10]$. Rarely, hypersensitivity reactions may occur.

Prolonged administration of intranasal calcitonin can prevent postmenopausal bone loss and is also able to increase trabecular bone mass among patients presenting established osteoporosis [10,11]. It has been demonstrated that the prolonged daily administration of 200 IU intranasal calcitonin inhibits bone resorption, increases lumbar spine BMD by $1.7 \%-3.3 \%$ after one year, and reduces vertebral fracture risk $[9,12]$. Furthermore, specific modification of bone microstructure was demonstrated at the distal radius using magnetic resonance imaging [13].
A possible effect of calcitonin on bone microarchitecture could be investigated using high-resolution p-QCT. By assessing structural bone changes, the present study should thus provide new insights in the mode of action of nasal calcitonin. We hypothezise that - compared to placebo - calcitonin improves or maintains microstructural parameters, with a possible difference between weight bearing and non-weight bearing bones.

\section{Methods \\ Study design}

This is a monocentric, randomized, placebo-controlled, double-blind trial over two years, assessing the effects of nasal calcitonin (200 IU/day) on bone microarchitecture measured by high resolution p-QCT in postmenopausal women.

This protocol was initiated by the principal investigator and has been accepted by the ethics committee of the University Hospitals of Geneva and by the Swiss legislative authority (Swissmedic). It has been registered in a public clinical trial data base [ClinicalTrials.gov number NCT00372099] [14].

\section{End points}

Primary efficacy endpoint will be changes in bone structure and bone microarchitecture evaluated by high resolution p-QCT in postmenopausal women compared to placebo during two years.

As a secondary endpoint we will investigate changes in markers of bone turnover.

\section{Study population}

The study population will consist of 100 postmenopausal women aged 45 to 70 years with a BMD T-Score between 0.0 and -2.49. In- and exclusion criteria are detailed in Table 1. Given the possible administration of placebo treatment, women with osteoporosis are excluded from the study for ethical reasons. Study participants are being recruited through advertisements in local newspapers; recruitment has started in January 2007.

\section{Study medication}

Participants receive 200 IU calcitonin nasal spray once daily or the matching placebo spray, provided by Novartis Pharmaceuticals, Basel, Switzerland. All patients fulfilling the inclusion/exclusion criteria will be given study medication. Odds of receiving active treatment or placebo are $1: 1$. Medication labels comply with the legal requirements in Switzerland and will be printed in the local language. The storage conditions for the study drug as well as the randomization number are specified on the medication label. No patient identifier is stated on the label. 
Table I: In- and exclusion criteria

Inclusion criteria

- Women aged 45 to70 years

- Natural or surgical menopause $=1$ year

- T-Score between 0.0 and -2.49 (spine or proximal femur - total or femoral neck, measured by DXA)

\section{Exclusion criteria}

- Osteoporosis (T-score $=-2.5$ in spine or proximal femur - total or femoral neck, measured by DXA)

- Severe vertebral fracture, as identified by screening DXA assessment

- Any history of metabolic disease that could affect bone metabolism: hyperparathyroidism, osteogenesis imperfecta, Paget's disease, osteomalacia

- Thyroid disease (if receiving thyroid hormone replacement, the patients must be euthyroid and on a stable dose of thyroid hormone)

- Impaired renal function (estimated creatinine clearance $<30 \mathrm{ml} / \mathrm{min}$ )

- History of previous or active malignancy of any organ system, treated or untreated, within the past 5 years.

- History of corticosteroid treatments during 6 months or more, daily dosage $>5 \mathrm{mg}$

- $\mathrm{BMI}<18$ or $>30 \mathrm{~kg} / \mathrm{m}^{2}$

- Treatments with estrogens, SERMs, tibolone, calcitonin, strontium ranelate, teriparatide or PTH, or oral bisphosphonates in the previous year, if duration $>2$ weeks. Treatment with iv bisphosphonates or iv calcitonin of any duration in the previous year

All patients receive a calcium and vitamin $\mathrm{D}$ supplement $(1000 \mathrm{mg} / 880 \mathrm{UI}$ per day). If the supplement is not tolerated, the dose will be reduced or, if necessary, the supplement will be stopped. In this case, the participant may continue the trial and will be given advice on adequate dietary calcium intake. A vitamin D supplement of 800 $\mathrm{IU} /$ day will then be prescribed during the winter months.

Drug accountability will be noted during visits and at the completion of the trial. Patients will be asked to return all unused medication at the end of the study.

\section{Blinding}

Patients, investigator staff, persons performing the assessments, and data analysts will remain blind to the identity of the treatment from the time of randomization until database lock. The identity of the treatments will be concealed by the use of study drugs that are all identical in packaging, labeling, schedule of administration, appearance, taste, and odor. Randomization data are kept strictly confidential until the time of unblinding and will not be accessible by anyone else involved in the study. Unblinding will only occur in the case of patient emergencies and at the conclusion of the study.

\section{Study procedures}

Potential participants will undergo a screening procedure, including recording of medical history, physical examination, blood sampling, and BMD measurements and vertebral fracture assessment (VFA) by DXA (Prodigy, GE

Table 2: Visit schedule

\begin{tabular}{|c|c|c|c|c|c|c|}
\hline & Visit I & Visit 2 & Visit 3 & Visit 4 & Visit 5 & Visit 6 \\
\hline Period & Screening & Baseline & Month 6 & Month 12 & Month 18 & Month 24 \\
\hline Day & -7 to -3 & 0 & $180 \pm 14$ & $360 \pm 14$ & $540 \pm 14$ & $720 \pm 14$ \\
\hline Informed consent & $x$ & & & & & \\
\hline In-/exclusion criteria & $x$ & $\mathrm{x}$ & & & & \\
\hline Medical history & $x$ & $\mathrm{x}$ & & & & \\
\hline Prior/Concomitant medication & $x$ & $\mathrm{x}$ & $\mathrm{x}$ & $\mathrm{x}$ & $\mathrm{x}$ & $\mathrm{x}$ \\
\hline Vital signs & & $x$ & & $x$ & & $x$ \\
\hline Physical examination & $x$ & & & & & $x$ \\
\hline Adverse events & & & $\mathrm{x}$ & $x$ & $x$ & $x$ \\
\hline DXA & $x$ & & & $\mathrm{x}$ & & $x$ \\
\hline PQCT & & $\mathrm{x}$ & & $\mathrm{x}$ & & $x$ \\
\hline Laboratory test & $x$ & & & $x$ & & $x$ \\
\hline Bone markers & $x$ & $x$ & $x$ & $x$ & $x$ & $x$ \\
\hline Antibody test & & & & & & $x$ \\
\hline Study drug dispensing & & $\mathrm{x}$ & $\mathrm{x}$ & $x$ & $x$ & \\
\hline Drug accountability & & & $\mathrm{x}$ & $x$ & $\mathrm{x}$ & $x$ \\
\hline Study termination sheet & & & & & & $x$ \\
\hline
\end{tabular}


Healthcare Technologies, Waltham, Wisconsin, USA). Participants fulfilling the inclusion/exclusion criteria at screening will be included in the study. During the study, visits to the trial center will take place every 6 months (Table 2).

\section{Efficacy assessments}

Bone microarchictecture measurements

Bone microarchitecture will be investigated by p-QCT at baseline and after one and two years. Three-dimensional high-resolution measurements will be performed at the distal radius and tibia using an Xtreme CT device (Scanco Medical AG, Bassersdorf, Switzerland), as described by others [15]. In brief, the non-dominant limb (or the nonfractured limb in case of prior fractures) will be scanned at the standardized locations for human in vivo measurements. The following settings are used: effective energy of $60 \mathrm{kVp}$, matrix size $1536 \times 1536$, x-ray tube current 900 $\mu \mathrm{A}$, slice increment $82 \mu \mathrm{m}$. One hundred and ten slices will be obtained with a voxel size of $82 \mu \mathrm{m}$. The effective dose for one Xtreme CT standard measurement is $3 \mu \mathrm{Sv}$ per site. Processing of the obtained scans will be done in accordance with the default protocol of the device. Table 3 lists the assessed bone parameters. In our institution, the in-vivo coefficient of variation $(\mathrm{CV} \%)$ for these bone indices ranges from $0.5-1 \%$ (vol. densities) to $3-5 \%$ (TbN). Quality control is performed on a daily basis.

\section{Bone mineral density measurements}

Spine and hip bone mineral density will be measured at the baseline visit and after one and two years by DXA (Prodigy, GE Healthcare Technologies, Wisconsin, USA). The irradiation dose is $7 \mu \mathrm{Sv}$ per examination.

\section{Laboratory test}

Fasting serum samples will be obtained from all patients at the 6 -monthly visits. Serum will be stored at $-70^{\circ} \mathrm{C}$ to

Table 3: Parameters of bone microarchitecture

\begin{tabular}{l}
\hline Bone densities \\
\hline - cortical bone density (Dcort; $\left.\mathrm{mgHA} / \mathrm{cm}^{3}\right)$ \\
- trabecular bone density (Dtrab; $\left.\mathrm{mgHA} / \mathrm{cm}^{3}\right)$ \\
- meta trabecular bone density $\left(\mathrm{Dmeta} ; \mathrm{mgHA} / \mathrm{cm}^{3}\right)$ \\
- inner trabecular bone density $\left(\mathrm{Dinn} ; \mathrm{mgHA} / \mathrm{cm}^{3}\right)$ \\
- average bone density (Dtot; $\left.\mathrm{mgHA} / \mathrm{cm}^{3}\right)$ \\
\hline Bone structure \\
\hline - trabecular bone volume to tissue volume (BV/TV; \%) \\
- number of trabeculae (Tb.N; $\left.\mathrm{mm}^{-1}\right)$ \\
- trabecular thickness (Tb.Th; $\mu \mathrm{m})$ \\
- trabecular separation (Tb. Sp; $\mu \mathrm{m}$ ) \\
- cortical thickness (C.Th; $\mu \mathrm{m})$ \\
- index of the inhomogeneity of the network (Tb. I/N.SD) \\
- cross-sectional area (CSA; mm ${ }^{2}$ ) \\
\hline
\end{tabular}

allow analysis in batches. Serum total procollagen type 1 amino-terminal propeptide (P1NP), a marker of bone formation, and serum C-terminal telopeptide of type I collagen ( $\beta$-crosslaps, CTX), a marker of bone resorption, will both be measured using electrochemiluminescence immunoassays (Elecsys 2010, Roche Diagnostics). The intrassay coefficients of variation $(\mathrm{CV} \%)$ for these measurements are 2.3-3.7\% and 1.6-4.7\%, respectively. Levels of serum PTH (7-84) will also be assessed using an electrochemiluminescence immunoassay (CV 4.3-7.1\%); serum 25-hydroxyvitamin $\mathrm{D}$ will be determined by a competitive immunoassay (ELISA; CV 5.3-6.7\%).

Serum and urine biochemistry as well as hematology will be measured immediately after blood sampling in the routine laboratory according to standard procedures. The titer of calcitonin antibodies will be assessed at end of the study.

\section{Safety assessments}

Safety assessments will include the monitoring and recording of all adverse events (AEs), including serious adverse events (SAEs), the regular monitoring of hematology, blood chemistry and urine values, regular monitoring of vital signs, physical condition and body weight. Body height will be measured 6-monthly with a Harpeden stadiometer (Holtain Ltd, Crymych, UK).

\section{Data management}

All original data will be recorded in the patient's source documentation. Subsequently, anonymized data will be entered into an electronic database and displayed in the patient data listings. Tables will display counts of missing values. Quality control of the data will be done at regular intervals, using descriptive statistics and visual analyses to check for inconsistencies and extreme values. Detected errors will be rectified according to the source data. Data will be handled in accordance with the good clincal practice guidelines and applicable local regulations. Data management and analysis as well as interpretation and publication of the results remain under the responsibility of the principal investigator and will be performed by the study team of the University Hospitals of Geneva.

\section{Statistical methods}

\section{Sample size calculation}

A total number of 80 patients (40 in the calcitonin group and 40 in the control group) is necessary to have a statistical power of $80 \%$ to detect an expected difference of $3.5 \%$ in BV/TV [13]. Due to an expected drop-out rate of $20 \%$, a total of 100 patients should be enrolled.

\section{Statistical analysis}

Data will be summarized with respect to demographic and baseline characteristics, efficacy observations and 
measurements, and safety observations and measurements.

To assess the treatment effects of calcitonin on bone microarchitecture and provide a proof of concept, the primary analysis of the efficacy endpoints in this exploratory trial will be based on the observed data of the per-protocol population. Secondarily, an ITT analysis may be performed to test the robustness of the results.

For each analyzed parameter a two-sided t-test will be applied. The change from baseline between the two different groups (active treatment/placebo) will be compared by an analysis of covariance (ANCOVA) model with change as the response variable, the baseline value as a covariate, and treatment as a fixed factor.

The assessment of safety will be based mainly on the frequency of adverse events and on the number of laboratory values that fall outside of pre-determined ranges. Other safety data (e.g. electrocardiogram, vital signs, special tests) will be considered as appropriate.

Analysis of adverse events will be based on the safety population which includes all randomized patients who have taken at least one dose of the study drug. Adverse events will be summarized by presenting, for each treatment group, the number and percentage of patients having any adverse event, having an adverse event in each body system and having each individual adverse event. Any other information collected (e.g. severity or relatedness to study medication) will be listed as appropriate.

\section{Summary}

This study will investigate the effects of nasal salmon calcitonin on bone microarchitecture and its relation with markers of bone turnover in postmenopausal women. It should thus give a new insight into the specific effects of calcitonin on different bone parameters.

\section{Competing interests}

M.E. and M.A. are employees of Novartis. All other authors declare that they have no competing interests. This study is financially supported by Novartis.

\section{Authors' contributions}

LR participated in the design and coordination of the study, was responsible for the data acquisition and management, and drafted the manuscript. BU contributed to study coordination and clinical care of the trial participants. ME and MA provided useful advice for the study design. RR is the initiator and principal investigator of the study and drafted the original protocol. All authors read and approved the final manuscript.

\section{Acknowledgements}

This study is financially supported by Novartis. We thank Christine Strohmaier, Katharina Gasser and Andrea Dach from Novartis for constructive discussions and the provision of the study drugs; François R. Herrmann and Raphael Grandjean for the creation of the database; and Anne Sigaud for taking care of the study subjects.

\section{References}

I. Turner $\mathrm{CH}$ : Biomechanics of bone: determinants of skeletal fragility and bone quality. Osteoporosis Int 2002, I3(2):97- 104.

2. Ammann P, Rizzoli R: Bone strength and its determinants. Osteoporosis Int 2003, 14(Suppl 3):13-18.

3. Seeman E, Delmas PD: Bone quality-the material and structural basis of bone strength and fragility. New EnglJ Med 2006, 354:2250-2261.

4. Garnero P, Hausherr E, Chapuy MC, Marcelli C, Grandjean H, Muller C, Cormier C, Breart G, Meunier PJ, Delmas PD: Markers of bone resorption predict hip fracture in elderly women: the EPIDOS Prospective Study. J Bone Miner Res 1996, I I:153 I- 1538.

5. Laib A, Ruegsegger P: Calibration of trabecular bone structure measurements of in vivo three-dimensional peripheral quantitative computed tomography with 28-microm-resolution microcomputed tomography. Bone 1999, 24:35-39.

6. Müller R, Hahn M, Vogel M, Delling G, Rüegsegger P: Morphometric analysis of noninvasively assessed bone biopsies: comparison of high-resolution computed tomography and histologic sections. Bone 1996, 18:215-220.

7. Boonen S, Cheng XG, Nijs J, Nicholson PH, Verbeke G, Lesaffre E, Aerssens J, Dequeker J: Factors associated with cortical and trabecular bone loss as quantified by peripheral computed tomography (pQCT) at the ultradistal radius in aging women. Calcif Tissue Int 1997, 60: 164-170.

8. Rüegsegger $P$, Durand $E P$, Dambacher MA: Differential effects of aging and disease on trabecular and compact bone density of the radius. Bone $|99|, \mid$ | 2:99- 105.

9. Chesnut $\mathrm{CH}$ 3rd, Silverman S, Andriano K, Genant H, Gimona A, Harris S, Kiel D, LeBoff M, Maricic M, Miller P, Moniz C, Peacock M, Richardson P, Watts N, Baylink D: A randomized trial of nasal spray salmon calcitonin in postmenopausal women with established osteoporosis : the prevent recurrence of osteoporotic fractures study. PROOF Study Group. Am J Med 2000, 109:267-276.

10. Overgaard K, Hansen MA, Jensen SB, Christiansen C: Effect of salcatonin given intranasally on bone mass and fracture rates in established osteoporosis: a dose-response study. BMJ 1992 , 305:556-56I.

II. Gennari C, Agnusdei D, Montagnani M, Gonnelli S, Civitelli R: An effective regimen of intranasal salmon calcitonin in early postmenopausal bone loss. Calcif Tissue Int 1992, 50:38I-383.

12. Thamsborg G, Jensen JE, Kollerup G, Hauge EM, Melsen F, Sorensen $\mathrm{OH}$ : Effect of nasal salmon calcitonin on bone remodeling and bone mass in postmenopausal osteoporosis. Bone 1996, I8:207-2| 2 .

13. Chesnut CH 3rd, Majumdar S, Newitt DC, Shields A, Van Pelt J, Laschansky E, Azria M, Kriegman A, Olson M, Eriksen EF, Mindeholm L: Effects of salmon calcitonin on trabecular microarchitecture as determined by magnetic resonance imaging: results from the QUEST study. J Bone Miner Res 2005, 20: I548-I56I.

14. University Hospital Geneva: Bone microstructure in nasal salmon calcitonin treated postmenopausal women (a pilot study). ClinicalTrials.gov [Internet] 2006 [http://clinicaltrials.gov/ct/ show/NCT00372099 NLM Identifier: NCT00372099]. Bethesda (MD): National Library of Medicine (US) cited 2007 Sep 05

15. Boutroy S, Bouxsein ML, Munoz F, Delmas PD: In vivo assessment of trabecular bone microarchitecture by high-resolution peripheral quantitative computed tomography. J Clin Endocrinol Metab 2005, 90:6508-65I5. 